Pacific

Journal of

Mathematics

ACTIONS OF LINEAR ALGEBRAIC GROUPS OF EXCEPTIONAL TYPE ON PROJECTIVE VARIETIES

KiWAMU WATANABE

Volume $239 \quad$ No. 2

February 2009 


\title{
ACTIONS OF LINEAR ALGEBRAIC GROUPS OF EXCEPTIONAL TYPE ON PROJECTIVE VARIETIES
}

\author{
KIWAMU WATANABE
}

\begin{abstract}
Let $X$ be a smooth projective variety of dimension $n$ and $G$ a simple linear algebraic group of exceptional type acting regularly and nontrivially on $X$. Then it is known that $n$ has a lower bound $r_{G}$ which only depends on the Dynkin type of $G$. In this article we give a classification of $X$ with an action of $G$ in the case where $n=r_{G}+1$.
\end{abstract}

\section{Introduction}

Let $X$ be a smooth projective variety of dimension $n$ and $r_{G}$ the minimum of the dimension of a homogeneous variety of a simple linear algebraic group $G$, that is, the minimum codimension of a maximal parabolic subgroup of $G$. M. Andreatta [2001] proved that if $r_{G}<n$, the only regular action of $G$ on $X$ is trivial, and if $r_{G}=n$, then $X$ is homogeneous. He also gave a classification of smooth projective varieties on which a simple linear algebraic group of classical type acts regularly and nontrivially in the case where $n=r_{G}+1$. Our main purpose of this article is to prove the following:

Theorem 1.1. Let $X$ be a smooth projective variety of dimension $n$ and $G$ a simple, simply connected and connected linear algebraic group of exceptional type acting regularly and nontrivially on $X$. Assume that $n=r_{G}+1$. Then $X$ is one of the following; the action of $G$ is unique for each case:

(i) $\mathbb{P}^{6}$,

(ii) $\mathbb{Q}^{6}$,

(iii) $E_{6}\left(\omega_{1}\right)$,

(iv) $G_{2}\left(\omega_{1}+\omega_{2}\right)$,

(v) $Y \times Z$, where $Y$ is $E_{6}\left(\omega_{1}\right), E_{7}\left(\omega_{1}\right), E_{8}\left(\omega_{1}\right), F_{4}\left(\omega_{1}\right), F_{4}\left(\omega_{4}\right), G_{2}\left(\omega_{1}\right)$ or $G_{2}\left(\omega_{2}\right)$, and $Z$ is a smooth projective curve,

(vi) $\mathbb{P}\left(\mathscr{O}_{Y} \oplus \mathcal{O}_{Y}(m)\right)$, where $Y$ is as in $(\mathrm{v})$ and $m>0$.

MSC2000: primary 14L30, 14L40; secondary 14E30.

Keywords: group action, linear algebraic group of exceptional type, minimal model program.

Supported by Research Fellowships of the Japan Society for the Promotion of Science for Young Scientists. 
Note that $G$-orbits on $X$ are very simple (for example a projective space and a quadric) in the case where $G$ is classical type, but they are not in our case. So we need other arguments than Andreatta's in several points.

Throughout this paper we work over the complex number field $\mathbb{C}$.

\section{Preliminaries}

We denote a simple linear algebraic group of Dynkin type $G$ simply by $G$ and for a dominant integral weight $\omega$ of $G$, the minimal closed orbit of $G$ in $\mathbb{P}\left(V_{\omega}\right)$ by $G(\omega)$, where $V_{\omega}$ is the irreducible representation space of $G$ with highest weight $\omega$. For example, $E_{6}\left(\omega_{1}\right)$ is the minimal closed orbit of an algebraic group of type $E_{6}$ in $\mathbb{P}\left(V_{\omega_{1}}\right)$, where $\omega_{1}$ is the first fundamental dominant weight in the standard notation of Bourbaki [1968]. Then we call $G(\omega)$ a rational homogeneous variety.

Lemma 2.1 [Andreatta 2001, Lemmas 1.4, 1.5]. Let $X$ be a smooth projective variety on which a connected linear algebraic group $G$ acts regularly and nontrivially. Then $X$ has an extremal contraction $\phi: X \rightarrow Z$ which is $G$-equivariant, and $G$ acts regularly on $Z$.

Definition 2.2 [Andreatta 2001, Definition 1.8]. Let $G$ be a simple linear algebraic group. We define $r_{G}$ to be the minimal codimension of parabolic subgroups of $G$.

Example 2.3 [Andreatta 2001, Example 1.0.1]. If $G$ is an exceptional linear algebraic group, we have $r_{E_{6}}=16, r_{E_{7}}=27, r_{E_{8}}=57, r_{F_{4}}=15$ and $r_{G_{2}}=5$.

Proposition 2.4 [Andreatta 2001, Proposition 2.1]. Suppose that a connected reductive linear algebraic group $G$ acts effectively on a complete normal variety $Z$. Then the following are equivalent:

(1) There exists a fixed point $z$ such that its projectivized tangent cone, that is the variety $P_{z}=\operatorname{Proj}\left(\bigoplus_{k} m_{z}^{k} / m_{z}^{k+1}\right)$, is a G-homogeneous variety.

(2) $Z$ is a projective quasihomogeneous cone over a homogeneous variety with respect to $G$.

Proposition 2.5 [Andreatta 2001, Lemma 2.2 and Proposition 3.1]. Let $X$ be $a$ smooth projective variety of dimension $n$ and $G$ a simple, simply connected, connected linear algebraic group acting regularly and nontrivially on $X$. Then

(1) $n \geq r_{G}$;

(2) if moreover $n=r_{G}$, then $X$ is homogeneous;

(3) if $G$ is exceptional and $n=r_{G}+1, X$ has no fixed points.

Lemma 2.6 [Andreatta 2001, Lemma 4.2]. Let $X$ and $Y$ be smooth projective varieties on which a simple exceptional linear algebraic group $G$ acts regularly and nontrivially. Assume that $r_{G}=\operatorname{dim} X-1=\operatorname{dim} Y-1$. If $X$ and $Y$ each have a dense open orbit which is $G$-isomorphic, then we have a $G$-isomorphism $X \cong Y$. 
Proposition 2.7 [Watanabe 2008]. Let $X$ be a smooth projective variety and $A$ a rational homogeneous variety $G(\omega)$, where $G$ is exceptional. If $A$ is an ample divisor on $X,(X, A)$ is isomorphic to $\left(\mathbb{P}^{6}, \mathbb{Q}^{5}\right),\left(\mathbb{Q}^{6}, \mathbb{Q}^{5}\right)$ or $\left(E_{6}\left(\omega_{1}\right), F_{4}\left(\omega_{4}\right)\right)$.

Remark that a 5 -dimensional smooth quadric $\mathbb{Q}^{5}$ is $G_{2}$-homogeneous.

\section{Proof of Theorem 1.1}

By Lemma 2.1 we have a $G$-equivariant extremal contraction of a ray $\phi: X \rightarrow Z$. Assume that $\rho(X) \geq 2$.

Case 1. $\phi$ is birational. Let $\phi$ be birational and $E$ the exceptional locus of $\phi$. Since $r_{G}$ is equal to $n-1$ and $X$ has no fixed points, $\phi$ is a divisorial contraction and $E$ is contracted to a point $z$. Furthermore $E$ is isomorphic to $E_{6}\left(\omega_{1}\right)\left(=E_{6}\left(\omega_{5}\right)\right)$, $E_{7}\left(\omega_{1}\right), E_{8}\left(\omega_{1}\right), F_{4}\left(\omega_{1}\right), F_{4}\left(\omega_{4}\right), G_{2}\left(\omega_{1}\right)\left(=Q^{5}\right)$ and $G_{2}\left(\omega_{2}\right)$. The conormal bundle of the exceptional divisor is $N_{E / X}{ }^{*} \cong \mathcal{O}(k)$ with $1 \leq k \leq i(E)-1$, where $i(E)$ is the Fano index of $E$.

Applying Proposition 2.4, we see that $X$ is a completion of an open orbit $G / K$ (see [Ahiezer 1977]). Here $K$ is the kernel of the character map $\rho: P \rightarrow \mathbb{C}^{*}$ associated to the homogeneous line bundle $N_{E / X}{ }^{*} \cong \mathcal{O}(k)$, where $P$ is the parabolic subgroup which satisfies $E \cong G / P$.

On the other hand, $X_{k}=\mathbb{P}\left(N_{E / X}{ }^{*} \oplus \mathcal{O}\right)$ is also a completion of an open orbit $G / K$. By Lemma 2.6, $X$ is isomorphic to $X_{k}=\mathbb{P}\left(N_{E / X}{ }^{*} \oplus 0\right)$.

Case 2. $\phi$ is a fibering type. Let $\phi$ be a contraction of fibering type.

First we assume that the induced action of $G$ on $Z$ is trivial. In this case, any fiber of $\phi$ is isomorphic to $E_{6}\left(\omega_{1}\right), E_{7}\left(\omega_{1}\right), E_{8}\left(\omega_{1}\right), F_{4}\left(\omega_{1}\right), F_{4}\left(\omega_{4}\right), G_{2}\left(\omega_{1}\right)$ or $G_{2}\left(\omega_{2}\right)$ and $\operatorname{dim} Z=1$. Since rational homogeneous varieties are locally rigid, there is no $\phi$ which has both $F_{4}\left(\omega_{1}\right)$ and $F_{4}\left(\omega_{4}\right)$ (respectively $G_{2}\left(\omega_{1}\right)$ and $\left.G_{2}\left(\omega_{2}\right)\right)$ as fibers. So all fibers of $\phi$ are isomorphic to each other. Then we have $X=E_{6}\left(\omega_{1}\right) \times Z$, $E_{7}\left(\omega_{1}\right) \times Z, E_{8}\left(\omega_{1}\right) \times Z, F_{4}\left(\omega_{1}\right) \times Z, F_{4}\left(\omega_{4}\right) \times Z, G_{2}\left(\omega_{1}\right) \times Z$ or $G_{2}\left(\omega_{2}\right) \times Z$. This follows from [Mabuchi 1979, Theorem 1.2.1].

Second we assume that the induced action of $G$ on $Z$ is not trivial. Then $Z$ is isomorphic to $E_{6}\left(\omega_{1}\right), E_{7}\left(\omega_{1}\right), E_{8}\left(\omega_{1}\right), F_{4}\left(\omega_{1}\right), F_{4}\left(\omega_{4}\right), G_{2}\left(\omega_{1}\right)$ or $G_{2}\left(\omega_{2}\right)$. It follows that all fibers have dimension one. Moreover, all fibers of $\phi$ are isomorphic to each other. So $\phi$ is a conic bundle which fibers are isomorphic to $\mathbb{P}^{1}$. Since the Brauer group of $Z$ is trivial, $X$ is $\mathbb{P}(\mathscr{E})$ with $\mathscr{E}$ a rank 2 vector bundle on $Z$.

The assumption that $n=r_{G}+1$ implies that the dimension of any orbit of $G$ in $\mathbb{P}(\mathscr{E})$ is at least $n-1$. If $\mathbb{P}(\mathscr{E})$ is $G$-homogeneous, then $\mathbb{P}(\mathscr{E})$ has another natural fibration structure $\mathbb{P}(\mathscr{E}) \rightarrow Z^{\prime}$, where $Z^{\prime}$ is a $G$-homogeneous variety whose Picard number is 1 [Baston and Eastwood 1989, 2.4]. Since $\operatorname{dim} Z+1=\operatorname{dim} X>\operatorname{dim} Z^{\prime}$, $\left(Z, Z^{\prime}\right)\left(\right.$ or $\left.\left(Z^{\prime}, Z\right)\right)$ is $\left(E_{6}\left(\omega_{1}\right), E_{6}\left(\omega_{5}\right)\right),\left(F_{4}\left(\omega_{1}\right), F_{4}\left(\omega_{4}\right)\right)$ or $\left(G_{2}\left(\omega_{1}\right), G_{2}\left(\omega_{2}\right)\right)$ [Snow 1989, 9.3]. However, if $\left(Z, Z^{\prime}\right)$ is $\left(E_{6}\left(\omega_{1}\right), E_{6}\left(\omega_{5}\right)\right)$ or $\left(F_{4}\left(\omega_{1}\right), F_{4}\left(\omega_{4}\right)\right)$, 
the fiber of $\mathbb{P}(\mathscr{E}) \rightarrow Z$ is not $\mathbb{P}^{1}$. Hence $\left(Z, Z^{\prime}\right)$ is $\left(G_{2}\left(\omega_{1}\right), G_{2}\left(\omega_{2}\right)\right)$ and we have $\mathbb{P}(\mathscr{E}) \cong G_{2}\left(\omega_{1}+\omega_{2}\right)$.

If $\mathbb{P}(\mathscr{E})$ is not $G$-homogeneous, we have the $G$-orbit decomposition $\mathbb{P}(\mathscr{E})=$ $\left(\bigsqcup_{i \in I} G x_{i}\right)$ or $\mathbb{P}(\mathscr{E})=G x \sqcup\left(\bigsqcup_{i \in I} G x_{i}\right)$, where $x, x_{i} \in \mathbb{P}(\mathscr{E})$. Here, $G x$ is a $G$ orbit of dimension $n$ and $G x_{i}$ is a rational homogeneous variety of dimension $n-1$ whose Picard number is 1 . Since $\operatorname{dim} G x_{i}=\operatorname{dim} Z, \phi_{G x_{i}}: G x_{i} \rightarrow Z$ is a finite morphism. If the ramification divisor $R$ of $\phi_{G x_{i}}$ is not empty, $G$ acts on $R$. But this contradicts homogeneity of $G x_{i}$. So $\phi_{G x_{i}}$ is étale. Hence we see that $\phi_{G x_{i}}: G x_{i} \rightarrow Z$ is isomorphic, because a Fano variety is simply connected. So $G x_{i}$ is a section of $\phi$. Since any $G$-homogeneous vector bundle has no a transitive action of $G$, we have $\sharp I \neq 1$. So $\mathbb{P}(\mathscr{E})$ has two sections which do not intersect each other. Hence $\mathscr{E}$ is decomposable. The uniqueness of action can be proved as above.

Assume that $\rho(X)=1$. By using the list of parabolic subgroups of codimension $n$ corresponding to one node of the Dynkin diagram, we see that $X$ is not $G$ homogeneous. So $X$ has a closed orbit $H$ which is isomorphic to $E_{6}\left(\omega_{1}\right), E_{7}\left(\omega_{1}\right)$, $E_{8}\left(\omega_{1}\right), F_{4}\left(\omega_{1}\right), F_{4}\left(\omega_{4}\right), G_{2}\left(\omega_{1}\right)$ or $G_{2}\left(\omega_{2}\right)$. The condition $\rho(X)=1$ implies $X$ is a Fano variety. Furthermore, $\operatorname{Pic}(\mathrm{X}) \cong \mathbb{Z}$. Hence $H$ is an ample divisor of $X$. By Proposition 2.7 , we see that $(X, H)$ is $\left(\mathbb{P}^{6}, \mathbb{Q}^{5}\right),\left(\mathbb{Q}^{6}, \mathbb{Q}^{5}\right)$ or $\left(E_{6}\left(\omega_{1}\right), F_{4}\left(\omega_{4}\right)\right)$.

These $X$ satisfy the assumption of the Theorem. In fact, we see that $F_{4} \subset E_{6}$, $G_{2} \subset \mathrm{SO}(7) \subset \mathrm{SO}(8)$. Here $\mathrm{SO}(k)$ means the special orthogonal group.

At last, we shall prove the uniqueness of action. We only deal with the case where $X$ is $E_{6}\left(\omega_{1}\right)$. We can prove other cases as the same.

Let $V_{27}$ be the irreducible representation space of $E_{6}$ with highest weight $\omega_{1}$. Then $E_{6}$ acts on $V_{27}$. If $G$ whose Dynkin type is $F_{4}$ acts on $E_{6}\left(\omega_{1}\right)$, we obtain a 27-dimensional representation $G \rightarrow \mathrm{GL}\left(V_{27}\right)$. By the Weyl dimension theorem and our assumption, it is easy to see that $V_{27}$ is a direct sum of a 26-dimensional irreducible representation space $V_{26}$ and a 1-dimensional irreducible representation space $V_{1}$. Furthermore, we see that irreducible representations $G \rightarrow \operatorname{GL}\left(V_{26}\right)$ and $G \rightarrow \mathrm{GL}\left(V_{1}\right)$ are unique. This implies that the action of $G$ on $E_{6}\left(\omega_{1}\right)$ is unique.

\section{Acknowledgements}

The author would like to express his gratitude to his supervisor Professor Hajime Kaji for some useful advice.

\section{References}

[Ahiezer 1977] D. N. Ahiezer, "Dense orbits with two endpoints", Izv. Akad. Nauk SSSR Ser. Mat. 41:2 (1977), 308-324, 477. In Russian; translated in Math. USSR-Izv. 11 (1977), 293-307. MR 57 \#12537 Zbl 0378.14009 
[Andreatta 2001] M. Andreatta, "Actions of linear algebraic groups on projective manifolds and minimal model program", Osaka J. Math. 38:1 (2001), 151-166. MR 2002c:14075 Zbl 1054.14061

[Baston and Eastwood 1989] R. J. Baston and M. G. Eastwood, The Penrose transform: its interaction with representation theory, Oxford University Press, New York, 1989. MR 92j:32112 Zbl 0726.58004

[Bourbaki 1968] N. Bourbaki, Groupes et algèbres de Lie, Chapitres IV-VI, Actualités Scientifiques et Industrielles 1337, Hermann, Paris, 1968. MR 39 \#1590 Zbl 0186.33001

[Mabuchi 1979] T. Mabuchi, "On the classification of essentially effective SL(2; C) $\times$ SL(2; C)actions on algebraic threefolds", Osaka J. Math. 16:3 (1979), 727-744. MR 81k:14033a Zbl 0422. 14029

[Snow 1989] D. M. Snow, "Homogeneous vector bundles", pp. 193-205 in Group actions and invariant theory (Montreal, 1988), edited by A. Bailynicki-Birula, CMS Conf. Proc. 10, Amer. Math. Soc., Providence, RI, 1989. MR 90m:14051 Zbl 0701.14017

[Watanabe 2008] K. Watanabe, "Classification of polarized manifolds admitting homogeneous varieties as ample divisors”, Math. Ann. 342:3 (2008), 557-563. MR 2430990

Received September 4, 2008. Revised October 16, 2008.

KiWAMU WATANABE

WASEDA UNIVERSITY

DEPARTMENT OF MATHEMATICAL SCIENCES

SCHOOL OF SCIENCE AND ENGINEERING

4-1 Онкиво 3-СНоме

SHINJUKU-KU, TOKYO 169-8555

JAPAN

kiwamu0219@fuji.waseda.jp 